\title{
Management of obstructive sleep apnea syndrome in children with craniofacial malformation
}

\author{
Hans L.J. Hoeve ${ }^{\mathrm{a}, *}$, Koen F.M. Joosten ${ }^{\mathrm{b}}$, Sandra van den Berg ${ }^{\mathrm{b}}$ \\ a Department of Otorhinolaryngology, Sophia Children's Hospital, Erasmus Medical Center Rotterdam, P.O. Box 2060, \\ 3000 CB Rotterdam, The Netherlands \\ b Department of Pediatric Intensive Care, Sophia Children's Hospital, Erasmus Medical Center Rotterdam, \\ Rotterdam, The Netherlands
}

\begin{abstract}
Children with craniofacial syndromes, especially those with midfacial hypoplasia, micrognathia, or deformation of the cranial base, are frequently suffering from obstructive sleep apnea syndrome (OSAS). It is important to recognize this condition. Diagnostic methods and therapeutic developments are discussed. Experience with 31 patients in the Sophia Children's Hospital is presented. The majority of these infants suffered from moderate or severe OSAS. Treatment varied from symptomatic (e.g. continuous positive airway pressure) to curative. These therapies could often prevent a tracheotomy. Still more curative treatment options are needed. (C) 1999 Elsevier Science Ireland Ltd. All rights reserved.
\end{abstract}

Keywords: Airway obstruction; Endoscopy; Polysomnography

\section{Introduction}

Children with craniofacial syndromes are frequently suffering from obstructive sleep apnea syndrome (OSAS). It is important to recognize this condition. In this presentation the diagnostic and therapeutic possibilities are discussed.

OSAS is defined by the American Thoracic Society as a disorder of breathing during sleep, characterized by prolonged partial upper airway obstruction and/or intermittent complete obstruction that disrupts normal ventilation during sleep and normal sleep patterns [1]. The obstruction

\footnotetext{
* Corresponding author..

E-mail address: aukes@chis.azr.nl (H.L.J. Hoeve)
}

implies collapse of the oropharynx (or the supraglottis) because that part of the airway lacks a framework. The only dilating force is a neuromuscular mechanism, and that function is diminished during sleep.

Five degrees of airway obstruction are distinguished:

- Grade 1: snoring.

- Grade 2: impeded respiration, disturbed sleep, arousal, but no apnea, hypopnea or hypoxemia (upper airway resistance syndrome, UARS).

- Grade 3: apnea or hypopnea, disturbed sleep without hypoxemia (OSAS).

- Grade 4: as grade 3, but with hypoxemia (OSAS). 
- Grade 5, OSAS with right heart failure.

In mild OSAS two to five periods of apnea/hypopnea occur per hour, in moderate OSAS five to 24 , in severe OSAS $>24$.

The localization of the obstruction in the craniofacial syndromes varies. In Down syndrome, the upper airway is narrow and compromised by chronic respiratory infections. Other factors are macroglossy and muscular hypotony. In craniosynostosis syndromes-Crouzon, Apert, Pfeiffer, Saethre-Chotzen - deformation of the cranial base and maxillary hypoplasia result in obstruction of the nasal and nasopharyngeal airway. Syndromes with micrognathia, such as Treacher Collins, Pierre Robin, Goldenhar and many others, are characterized by obstruction at the hypopharyngeal level. Achondroplasia implies deformation of the cranial base.

\section{Diagnostics}

Symptoms are related to sleep-snoring, obstructive apnea, enuresis and arousal - or related to daytime - drowsiness, cognitive function disorder, failure to thrive. ENT (ear, nose and throat) examination of infants with craniofacial disorders and suspected OSAS should focus on micrognathia, macroglossia, maxillar hypoplasia, a narrow nose or pharynx, cleft palate, tonsillar enlargement, etc.

Polysomnography in indicated to confirm the diagnosis of OSAS, to establish the degree of OSAS and the need for treatment. Cardiology consultation is indicated to diagnose heart failure. The next step is endoscopy of the airway to study the dynamics of the obstruction during spontaneous breathing, and to localize the obstruction; this is important in choosing the type of treatment. Magnetic resonance imaging (MRI), computed tomography $(\mathrm{CT})$ scan and plain radiograms are of limited value, because they do not demonstrate the dynamics of respiration.

\section{Therapy}

Therapeutic options are oxygen, continuous positive airway pressure (CPAP) via a ventilation nose/mouth mask during sleep, decongestive or
Table 1

Diagnoses of 31 patients with OSAS and craniofacial malformation in Sophia Children's Hospital

$\begin{array}{lr}\text { Pierre Robin } & 7 \\ \text { Down } & 5 \\ \text { Micro/retrognathia } & 4 \\ \text { Nasal stenosis } & 4 \\ \text { Achondroplasia } & 1 \\ \text { Apert } & 1 \\ \text { Crouzon } & 1 \\ \text { Charge } & 1 \\ \text { Goldenhar } & 1 \\ \text { Hunter } & 1 \\ \text { Hurler } & 1 \\ \text { Noonan } & 1 \\ \text { Orofacial digit } & 1 \\ \text { Palatoschisis } & 1 \\ \text { Rubinstein Taybi } & 1\end{array}$

steroid nose drops, nasal surgery, surgery of choanal atresia, adenotonsillectomy, prone position, nasopharyngeal intubation, tongue surgery, anterior distraction of the maxilla or mandible and a tracheotomy.

\section{Patients}

At Sophia Children's Hospital 31 infants with craniofacial syndromes and airway obstruction were diagnosed and treated in the period 19941998 (Table 1). The degree of OSAS was established by means of polysomnography in the majority of cases (Table 2). Further diagnostic evaluation included radiological examination when indicated, and endoscopy of the airway. This resulted in curative treatment if possible, or symptomatic treatment. Many patients had more than one type of treatment. In Table 3 the most essential treatment per patient is presented.

Table 2

Degree of OSAS in 31 patients in the Sophia Children's Hospital

\begin{tabular}{lr}
\hline Obstruction, no OSAS & 2 \\
Mild OSAS & 5 \\
Moderate OSAS & 11 \\
Severe OSAS & 8 \\
Clinical OSAS, degree unknown & 5
\end{tabular}


Table 3

Therapy in 31 patients in Sophia Children's Hospital

\section{Curative therapy}

Adenotonsillectomy

Nose surgery and stents

Mandibular surgery

Removal of pharyngoplasty

Symptomatic therapy

Nose drops

Tracheotomy

Oxygen

Nasopharyngeal intubation

Prone positioning

CPAP

GE reflux medication

No therapy sidered unavoidable in many cases, this has been replaced gradually by less invasive or even curative therapies, as oxygen administration, CPAP, adenotonsillectomy, etc. The efficacy of these therapies can be monitored and adapted by means of polysomnography. This development has improved the quality of life in many infants. However, the majority of infants are still treated symptomatically because we still have too few curative possibilities. Future progress is to be found in developing more curative therapies, such as mandibular and maxillary surgery.

\section{References}

[1] American Thoracic Society, Standards and indications for cardiopulmonary sleep studies in children, Am. J. Respir. Crit. Care Med. 153 (1996) 866-878.

While in the recent past tracheostomy was con- 